\title{
What Affect True Information Findings from Rural Children Survey?
}

\author{
Abrar Noor Akramin Kamarudin, Bali Ranaivo-Malançon, and Nadianatra Musa \\ Department of Information System, Faculty of Computer Science and Information Technology, \\ Universiti Malaysia Sarawak \\ Kota Samarahan, Malaysia \\ min268@gmail.com,mbranaivo@unimas.my,nadia@unimas.my
}

\begin{abstract}
Recently, the Internet is accessible by the children in the rural area. The purpose of this paper is to understand what affects their true information findings based on the analysis of the survey. Data collection is obtained from the secondary school students $(\mathrm{N}=237)$ in Serian, a rural area of Sarawak. A self-administered questionnaire was conducted in three public secondary schools to obtain a socio-demographic profile, language usage, medium of assistance required, Internet access platforms and Internet content exposure. Logistic regression model tested the parent's educational level, Internet availability at home, English usage when online and mobile device easiness for learning in explaining the respondent's ability to find true information from the Internet. The focus group is able to obtain true information from the Internet if they are able to use English as their main Internet language as well as other factors. Based on the findings, an initial system framework is designed to personalize the children's Internet access.
\end{abstract}

Keywords - survey; personalization; Internet; children; rural area; true information findings; logistic regression

\section{INTRODUCTION}

Google.com is the most popular one-stop search engine among the netizens to find any information online. English, which is the main Internet language is not fully capable among the children who lived in the rural area of Sarawak. Besides the language barrier, limited telecommunication infrastructure inland might also contribute to the digital divide among them. Additionally, other two factors that contribute to the child's ability to find true information on the Internet can be their parent's educational background and inclination to selflearning by using a smartphone.

Imagine if Sarawak indigenous languages are supported by Google, another issue faced by the rural children is to obtain help on the Internet. Normally, people will type in any question into the search box to get some answers or at least ask for help from the others. However, these rural youngsters might not susceptible to identify the true and accurate information when they are presented with many options. For example, when a child needs to find an article on science subject, focusing on the pregnancy, the result is often mixed as illustrated in Fig. 1. Unfortunately, some web pages are obviously not appropriate for the child's view, although the SafeSearch feature is turned on. Thus, they are vulnerable to many unpleasant things if they are not supervised by the adult. The idea of this paper is to understand what affects true information findings from the Internet among the rural children, which further extended to design a personalized Internet access framework for them.

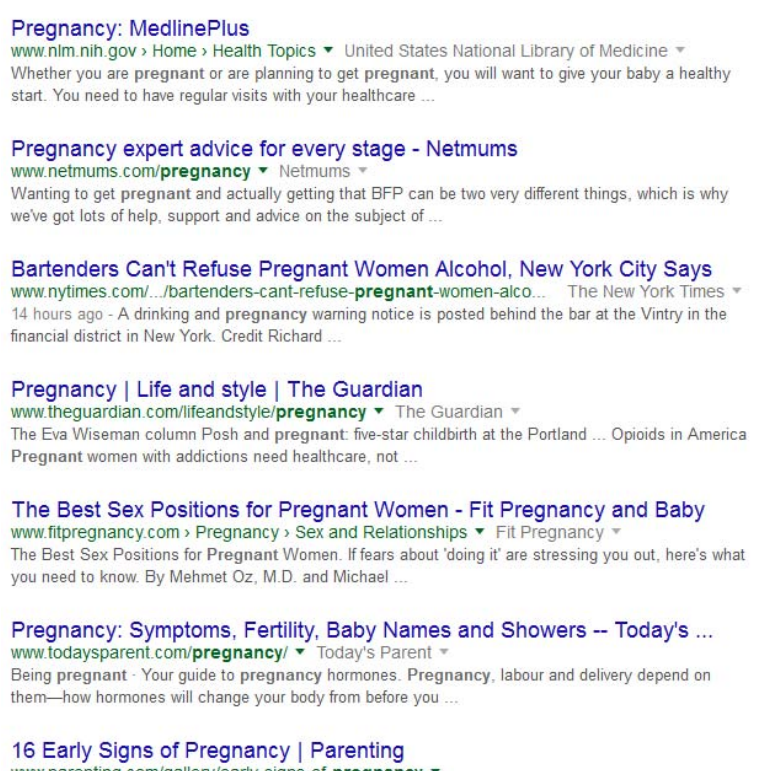

Fig. 1. Mixed search results from Google

\section{BACKGROUND}

Internet technology allows the access to more than 4.85 billion Web pages, the figure is indicated by the WorldWideWebSize.com on February 2016. Many of these web pages should not be viewed by children. The threats and risks related to the Internet are a fact, even though, "The Internet can harm, but can also be a child's best tool for learning" said the title of The Guardian on November 2013. In November 2014, while writing for the Foreword section of the document reporting the desk review conducted by UNICEF Malaysia, the Chairman of the Malaysian Communications and Multimedia Commission (MCMC) wrote: "Whilst Malaysians are enjoying the benefits from online services everywhere across the country, the MCMC is cognizant of the increasing need to safeguard them, especially children who are the most 